\title{
Frailty and neuropathology in relation to dementia status: the Cambridge City over-75s Cohort study
}

\author{
Lindsay Wallace, ${ }^{1,2}$ Sally Hunter, ${ }^{3}$ Olga Theou, 1,4 Jane Fleming, ${ }^{3}$ \\ Kenneth Rockwood, $1, *$ (i) and Carol Brayne $3, *$ \\ ${ }^{1}$ Department of Medicine, Dalhousie University, Halifax, Canada \\ ${ }^{2}$ Faculty of Graduate Studies, Dalhousie University, Halifax, Canada \\ ${ }^{3}$ Department of Public Health and Primary Care, Cambridge Public Health, University of Cambridge, Cambridge, UK \\ ${ }^{4}$ School of Physiotherapy, Dalhousie University, Halifax, Canada
}

Objective: To examine the relative contributions of frailty and neuropathology to dementia expression in a population-based cohort study.

Design: Cross-sectional analysis of observational data.

Setting: Population-representative clinicopathological cohort study.

Participants: Adults aged $75+$ recruited from general practice registries in Cambridge, UK, in 1985.

Measurements: A 39-item frailty index and 15-item neuropathological index were used to operationalize frailty and neuropathology, respectively. Dementia status was ascertained by clinical consensus at time of death. Relationships were evaluated using logistic regression models in participants with autopsy records $(n=183)$. Model fit was assessed using change in deviance. Population attributable fraction for frailty was evaluated in relation to dementia incidence in a representative sample of the survey participants $(n=542)$.

Results: Participants with autopsy were $92.3 \pm 4.6$ years at time of death, and mostly women $(70 \%)$. Average frailty index value at last survey before death was $0.34 \pm 0.16$. People with dementia ( $63 \%$ of the sample) were frailer, had lower MMSE scores, and a higher burden of neuropathology. Frailty and neuropathological burden were significantly and independently associated with dementia status, without interaction; frailty explained an additional $3 \%$ of the variance in the model. Assuming a causal relationship and based on populationattributable fraction analyses, preventing severe frailty (Frailty Index $\geq 0.40$ ) could have avoided $14.2 \%$ of dementia cases in this population-based cohort.

Conclusions: In the very old, frailty contributes to the risk for dementia beyond its relationship with the burden of traditional dementia neuropathologies. Reducing frailty could have important implications for controlling the burden of dementia. Future research on frailty interventions should include dementia risk as a key outcome, public health interventions and policy decisions should consider frailty as a key risk factor for dementia, and biomedical research should focus on elucidating shared mechanisms of frailty and dementia development.

Key words: aging, dementia, frailty, neuropathology, Alzheimer's disease

Abbreviations: CC75C, Cambridge City over-75s Cohort study; SD, Standard Deviation; CAMDEX, Cambridge Mental Disorders of the Elderly Examination; DSM-IV, Diagnostic and Statistical Manual 4th Edition; MMSE, Multiple Mini State Examination; LATE-NC, Limbic-predominant Agerelated TDP-43 Encephalopathy-Neuropathologic Changes; AD, Alzheimer's disease; PAF, Population Attributable Fraction

\section{Background}

As treatments for clinically diagnosed Alzheimer's disease continue to fail in clinical trials, evidence is accumulating to suggest that diverse risk factors and

Correspondence should be addressed to: Carol Brayne, Cambridge Public Health, University of Cambridge, School of Clinical Medicine, Forvie Site, Cambridge Biomedical Cambus, Cambridge, CB2 OSR, UK. Phone: +44 1223330321. Email: cb105@medschl.cam.ac.uk. Received 30 Mar 2020; revision requested 02 Jul 2020; revised version received 14 Sep 2020; accepted 29 Oct 2020. First published online 15 February 2021

*Shared last authorship. mechanistic pathways are important, especially in late-life dementia (Canevelli et al., 2017). Many studies have now shown that single-protein abnormalities (e.g. plaques and tangles) are not highly correlated with the clinical expression of dementia, especially in the oldest old (Boyle et al., 2013; Brayne et al., 2009; Jansen et al., 2018; MRC CFAS, 2001; Wallace et al., 2019). The research paradigm for tackling dementia has assumed that Alzheimer's disease pathology is responsible for the majority of clinical expression of dementia (Jack et al., 2018), 
though increasingly evidence suggests that the majority of late-life dementia is associated with mixed pathology, including vascular abnormalities, and hippocampal sclerosis among others (Boyle et al., 2018; Wilson et al., 2010). In the general population, age remains the number one risk factor for dementia (Livingston et al., 2017), and understanding the context and contributions of aging in the development of dementia may provide insight into its complex etiology (Canevelli et al., 2015; Searle and Rockwood, 2015). Age-related diseases, such as heart disease and osteoarthritis, not only accumulate with age but also appear to be the result of small-scale (i.e. molecular) deficits which scale up to affect whole bodily systems in the form of frailty (Castell et al., 2015; Rockwood et al., 2015; Wallace et al., 2014). Internal or external insults are usually repaired easily by redundant repair mechanisms before becoming deficits, but as the body ages, the repair mechanisms fail and lead to the accumulation of deficits (Mitnitski et al., 2013; Mitnitski and Rockwood, 2015; Mitnitski et al., 2001).

Frailty is conceptualized as multisystem impairment giving rise to physiologic vulnerability to adverse health outcomes (Clegg et al., 2013) and is most commonly operationalized as a health state characterized by the accumulation of health deficits (Mitnitski et al., 2001), or as a phenotypic syndrome (Fried et al., 2001). Frailty is recognized as contributing to the dementia syndrome (Song et al., 2014; Sterniczuk et al., 2015), brain atrophy (Gallucci et al., 2018), mild cognitive impairment (Trebbastoni et al., 2017), cognitive decline (Thibeau et al., 2019) and predicts dementia incidence (Rogers et al., 2017). This evidence suggests that it is possible that the expression of dementia, even in the face of neuropathology, may be modified by frailty as measured by deficit accumulation (Anstey et al., 2014). Therefore, the objective of the current study was to examine the relative contributions of frailty and neuropathology to dementia expression in a population-based representative cohort study and to build on earlier work where frailty and neuropathology contributed independently to dementia risk in a sample of older adults in retirement homes (Wallace et al., 2020; 2019).

\section{Methods}

\section{Sample/participants}

The Cambridge City over-75s Cohort study was initiated in 1985 as a population representative sample (95\% response rate) of people aged 75 or above on general practice registers in Cambridge, $\mathrm{UK}$, including those living in care (Fleming et al.,
2007). It aimed to study cognition and function in older adults and enrolled 2610 participants of whom 2166 (all excluding one practice) were followed-up until their death (10 surveys over 28 years; see Additional Supplementary File 1 for study design). Each survey included questions on demographics, activities of daily living, and health problems. In cases where participants were unable to respond, proxy informants were sought. Early surveys were supplemented with additional CAMDEX (Cambridge Mental Disorders of the Elderly Examination) psychiatric assessments which included mental state examination, psychiatric history, performancebased cognitive testing, and a proxy informant interview. A brain donation program was initiated in survey 2 (year 2), and donation was agreed to and fulfilled by 242 participants, with known representation from the base population.

For the purpose of this cross-sectional study, we used the survey 3 ( 7 years after the initial survey) as our baseline as surveys from this wave on had the largest number of relevant variables to create a frailty index which could be used consistently across the remaining surveys.

At survey 3, 714 participants were interviewed, of whom 175 became brain donors; of these, 170 had complete neuropathological and frailty data, as well as known dementia status were included in the cross-sectional analysis. Some participants $(n=68)$ missed survey 3 but were followed-up in subsequent surveys and donated their brains, of these, $13 \mathrm{had}$ complete neuropathological and frailty data, and known dementia status, providing a total sample of 183 for cross-sectional analysis (Additional Supplementary File 2).

For the population-attributable risk analyses, we extended our inclusion criteria to participants who were not brain donors (as we were not assessing neuropathology, but rather the attributable risk of frailty to dementia). Here, we sampled the original 714 participants at survey 3 , excluded those with prevalent dementia $(n=153)$ or missing prevalence data at survey $3(n=2)$, and incomplete frailty data $(n=17)$ leaving us with a sample of 542 (Additional Supplementary File 2).

\section{Measures}

DEMENTIA STATUS

All clinical study records for brain donors were reviewed post-mortem and dementia status was ascertained by consensus by at least two clinicians using the Diagnostic and Statistical Manual 4th Edition (DSM-IV) criteria and blinded to neuropathological data (Brayne et al., 2009; Fleming et al., 2007). 
Frailty InDEX

The frailty index is a health state measure that reflects vulnerability to adverse health outcomes (Clegg et al., 2013). The frailty index $=$ (number of health deficits present)/(number of health deficits measured). For example, a person with 5 of 30 potential deficits measured has a frailty index score of $5 / 30=0.17$. Candidate variables from respondent interviews were indicative of poor health and included symptoms, signs, functional impairments, and comorbidities. These variables were screened against four criteria: (1) Relationship with age; (2) Prevalence of at least $1 \%$; (3) Less than $5 \%$ missing data across participants at any survey; (4) No more than $80 \%$ prevalence (saturation). A total of 39 items met all criteria and were included in the index. The index demonstrated properties consistent with frailty indices from similar samples (i.e. normal distribution with right skewed tail, higher frailty index in women than men, increase with age). As the goal of the regression analyses was to examine the cross-sectional relationship between frailty, neuropathology, and dementia, but neuropathology could only be obtained post-mortem via autopsy, we used a frailty index and dementia measurements obtained from participants' last survey prior to death. Baseline frailty index (survey 3) was used to predict population-attributable risk. Refer to Additional Supplementary File 3 for list of variables included in the frailty index. The frailty index was categorized into tertiles for the descriptive analyses, using cut-points of $0.27,0.43$, and a cut-point of 0.40 (corresponding to severely frail; Guaraldi et al., 2019) was used for the population-attributable risk analyses.

Neuropathological Index

Neuropathological data were obtained at autopsy by semi-quantitative scoring by trained neuropathologists according to the Consortium to Establish a Registry for Alzheimer's Disease (CERAD) protocol (Mirra, 1997; Mirra et al., 1991). Fifteen neuropathological variables were included in the index including: (1) atrophy, (2) pallor of substantia nigra and/or locus coerulus, (3) significant obstruction of large cerebral vessels, (4) gross parenchymal vascular lesions, (5) small vessel disease, (6) microinfarcts, (7) white matter pallor, (8) neuritic plaques, (9) amyloid deposits, (10) neurofibrillary tangles, (11) vascular amyloid, (12) granulovacuolar degeneration, (13) severe neuronal loss, (14) severe gliosis, (15) Lewy bodies. Other neuropathologies available in this cohort relating to Pick's disease, leukoencephalopathy, lobar atrophy, Creutzfeldt-Jakob disease, spongiform encephalopathy, neoplasms/tumors, Hirano bodies, ubiquitin, and Huntington's disease were not included in the index because they were absent in all cases. TDP-43 was excluded as there were too many missing cases. Full information on regional inclusions and coding are detailed in Additional Supplementary File 4. Included neuropathological variables were summed and divided by the number of valid variables (according to the deficit accumulation approach detailed above). The neuropathological index was then categorized into tertiles for the descriptive analyses, using cutpoints of $0.30,0.42$. Details of autopsy and neuropathological assessment can be found at cc75c .group.cam.ac.uk and Brayne et al. (2009).

\section{OTHER MEASURES}

Age, sex, education, post-mortem interval, and time from frailty evaluation to death were evaluated as confounders. Education (in years) was the only covariate found to be a significant independent predictor of the outcome, but age, sex, and education were conserved in final model for conceptual reasons.

\section{Statistical analysis}

Descriptive analysis techniques (including analysis of variance and $\chi^{2}$ tests) were used to describe the characteristics of the sample and assess distributions of frailty, neuropathology, and dementia in the sample.

Logistic regression models were used to explore the effect of frailty (frailty index) and neuropathology (neuropathological index) on dementia status independently and in the same model. Their interaction was also evaluated. Model fit was evaluated by change in deviance $\left(\chi^{2}\right.$ of -2 LogLikelihood values). Model assumptions including binary outcome, independent observations, no collinearity among independent variables, linearity of independent variables with log odds (according to Box Tidwell test), and large enough sample size based on least frequent outcome (Stoltzfus, 2011) were tested. Nagelkerke Pseudo $R^{2}$ was reported to show goodness of fit.

Complete-case analysis was employed, though 56 participants were excluded as they were missing more than $20 \%$ of the variables needed to calculate a frailty index. We undertook two sensitivity analyses to attempt to evaluate the effect of the missing data on our findings. First, we used an extreme case sensitivity analysis where missing frailty index values were allocated the highest level of frailty. Further, we attempted multiple imputations (chained equations algorithm) for frailty index values. 
Population-attributable fraction estimates the proportion of cases that hypothetically could be avoided if the exposure were eliminated or reduced. Here, we used this to determine the fraction of dementia cases (ascertained at time of death) that could be avoided if severe frailty (frailty index $\geq 0.40$ ) was "eliminated" or avoided at baseline. Populationattributable fraction was calculated using the following formula (Lin and Chen, 2019):

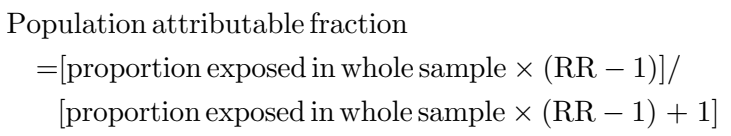

where $\mathrm{RR}=$ relative risk. As the exposure was rare, the hazard ratio approximates the relative risk and was used in order to control for time from baseline. By using a Cox proportional hazards model, we were able to control for time from baseline to death, as well as control for the contributions of baseline age, sex, and education. For this analysis, we extended our sample to include participants who did not have autopsy to achieve a larger and populationrepresentative sample. Statistical analyses were performed in SPSS version 25.0 and $\mathrm{R}$ version 3.5.2.

\section{Results}

Autopsy sample participants $(n=183)$ were aged 92.3 (SD 4.6; normally distributed) years on average at time of death, and mostly women (69.4\%). Average frailty index at last survey before death was 0.34 (SD 0.16; normally distributed). People with dementia were frailer, had lower Mini-Mental State Examination scores, and a higher burden of neuropathology (Table 1).

Very few participants demonstrated little to no neuropathology at death $(n=7 ; 4 \%)$. Among people with no dementia, $7.1 \%$ had a high burden of neuropathology. Among people with dementia, $16.3 \%$ demonstrated a low burden of neuropathology. Within each level of neuropathological burden, those with dementia generally had higher frailty (Figure 1), though dose-response was not consistent (likely due to small sample size).

The proportion of people with dementia was highest among people with a high frailty index and high burden of neuropathology, low among those with low frailty index and low burden of neuropathology, and in between for intermediate or either high frailty index or high neuropathology, suggesting these risk factors may be additive (Figure 1).

Logistic regression models satisfied assumptions and demonstrated that both frailty (at last survey before death) and neuropathological burden (at death) were significantly and independently associated with dementia status at last survey (Table 2), although they did not interact $(p=0.81)$. Addition of the frailty index to the model with the neuropathological index significantly improved model fit $X^{2}(1) 5.44, p<0.01$ ). Pseudo $\mathrm{R}^{2}$ increased from 0.085 to 0.123 suggesting that the addition of frailty to the model increased the explained variance by $3.8 \%$. Sensitivity analysis using extreme case imputation did not significantly change the results (Additional Supplementary File 5). Multiple Imputation by Chained Equations (MICE) was not used in this analysis as the only significant predictors of missing frailty index data were related to pathological measures that would be used in an interaction with the frailty index and would produce nonsensical results.

Based on a hazard ratio of 2.15 (95\% CI 1.01$4.60, p=0.048)$ and proportion of exposed (0.142) among 542 participants with no dementia at baseline (survey three), the $\mathrm{PAF}=0.142$, indicating that preventing severe frailty (frailty index $\geq 0.40$ ) on average 5.6 years before death would avoid $14.2 \%$ of dementia cases in this cohort.

Table 1. Descriptive characteristics of sample

\begin{tabular}{|c|c|c|c|}
\hline & $\begin{array}{l}\text { Whole sample } \\
\quad(n=183)\end{array}$ & $\begin{array}{l}\text { No dementia } \\
\quad(n=67)\end{array}$ & $\begin{array}{l}\text { Dementia } \\
(n=116)\end{array}$ \\
\hline Age at baseline (median; mean $\pm \mathrm{SD}$ ) & $85.2 ; 86.1 \pm 3.9$ & $85.4 ; 86.3 \pm 4.3$ & $85.0 ; 85.9 \pm 3.6$ \\
\hline Age at death (median; mean $\pm S D)$ & $92.1 ; 92.3 \pm 4.6^{\mathrm{a}}$ & $92.0 ; 92.3 \pm 4.8$ & $92.1 ; 92.4 \pm 4.4^{\mathrm{a}}$ \\
\hline $\operatorname{Sex}(n, \%$ female $)$ & $127(69.4 \%)$ & $44(65.7 \%)$ & $84(71.8 \%)$ \\
\hline Years of education (median; mean $\pm S D$ ) & $14.0 ; 15.3 \pm 2.4$ & $15.0 ; 15.8 \pm 2.6$ & $14.0 ; 15.0 \pm 2.2^{*}$ \\
\hline Years from last survey to death (median; mean \pm SD) & $1.8 ; 2.2 \pm 1.8$ & $1.8 ; 1.9 \pm 1.2$ & $2.0 ; 2.4 \pm 2.0$ \\
\hline MMSE at last survey before death (median; mean \pm SD) & $24.0 ; 21.4 \pm 6.6$ & $26.0 ; 25.7 \pm 3.1$ & $19.0 ; 18.7 \pm 6.8^{*}$ \\
\hline Frailty index (mean $\pm S D)$ & $0.34 \pm 0.16^{\mathrm{a}}$ & $0.30 \pm 0.13^{\mathrm{a}}$ & $0.36 \pm 0.17^{*, \mathrm{a}}$ \\
\hline Neuropathological index (mean \pm SD) & $0.37 \pm 0.13^{\mathrm{a}}$ & $0.34 \pm 0.14^{\mathrm{a}}$ & $0.39 \pm 0.13^{* a \mathrm{a}}$ \\
\hline
\end{tabular}

${ }^{a}$ Normally distributed.

${ }^{*} p<0.05$. 
Table 2. Logistic regression models for dementia status ( $n=183$; all models adjusted for age, sex, education) demonstrating that the frailty index and neuropathological index are independently associated with dementia status, even when included in the same model. Model fit is significantly improved when both frailty index and neuropathological index are included in a model for dementia status

\begin{tabular}{llrlrl}
\hline Model 1 & Frailty Index (per 0.1) & OR & $=1.33(95 \%$ CI 1.06-1.68), & & Deviance $=229.10$ \\
& & $p$ & $=0.015$ & & Nagelkerke $R^{2}=0.082$ \\
Model 2 & Neuropathological index (per 0.1) & OR & $=1.39(95 \%$ CI 1.06-1.81), & & Deviance $=227.75$ \\
& & $p$ & $=0.016$ & & Nagelkerke $R^{2}=0.085$ \\
Model 3 & Frailty Index (per 0.1) & OR & $=1.31(95 \%$ CI 1.04-1.66), & & Deviance $=222.31$ \\
& & & Nagelkerke $R^{2}=0.123$ \\
& & &
\end{tabular}

OR, Odds Ratio.

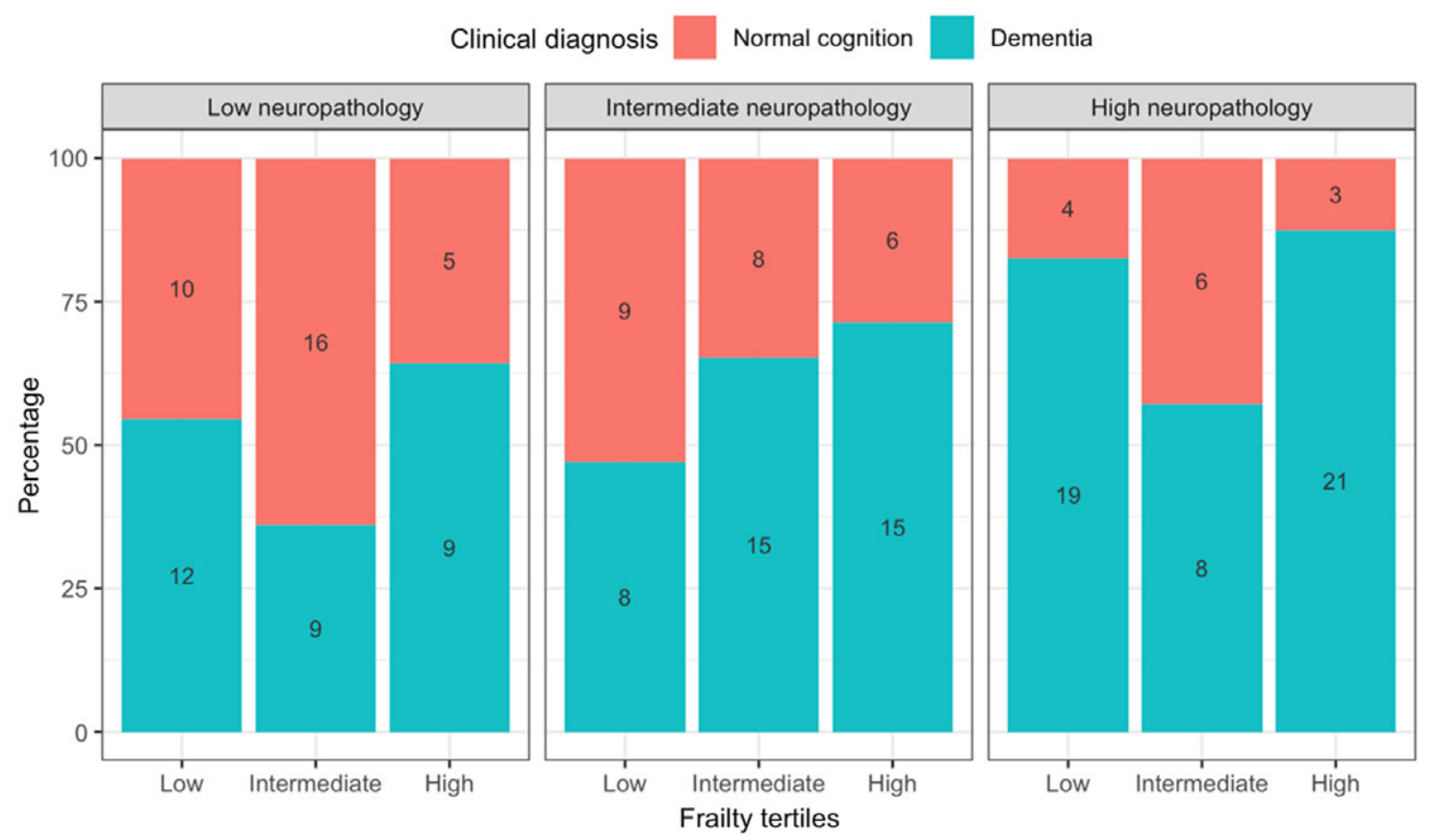

Figure 1. Proportion of participants with dementia according to tertiles of neuropathological index and frailty tertiles. Note: Frailty and dementia status were assessed at last survey before death (median 1.9 years pre-mortem), neuropathological burden was assessed at time of death. Numbers within the bars represent sample size.

\section{Discussion}

Almost a quarter of the sample $(23.4 \%)$ demonstrated a mismatch between neuropathological burden and clinical dementia (i.e. either dementia with low neuropathology or no dementia with high neuropathology), similar to previous reports (Brayne et al., 2009; Savva et al., 2009). Frailty explained additional variance and significantly predicted dementia status, even after controlling for the neuropathological index, but did not interact with the neuropathological index. Taken together, these results suggest that frailty and neuropathology may be additive risk factors that independently are neither necessary nor sufficient but are largely responsible for creating the conditions in which the clinical syndrome of dementia is experienced.
Given the independent risk conferred by frailty, we investigated what the scale of reduction of incidence of dementia would be, assuming a causal relationship. If this is the case, preventing severe frailty could reduce dementia risk by $14.2 \%$. This indicates that frailty treatment and management is a worthwhile area to focus on, not only in its own right but also for its consequences as part of societal attempts to reduce the impact of dementia in populations.

Although the sample was drawn from a population-representative cohort, those who participated in the autopsy subset (i.e. brain donors) were more likely to be diagnosed with dementia than those who did not have autopsy, as they were more likely to be followed-up and undergo clinical testing (see Additional Supplementary File 6 for comparisons). Nevertheless, the autopsy subset 
has shown to have cognitive score distributions that closely match those of the full cohort (Brayne et al., 2009; EClipSE Collaboration, 2009). The expected impact on our results here is minor, and likely reflects that frailty management would be slightly more important to control dementia in this group due to their other intersecting risks.

Another limitation was the sample size. While the sample size for the overall cohort study is quite large, by Survey 3 there had been significant attrition from this "older old" cohort due primarily to mortality, and missingness is to be expected as individuals become frail (Brayne et al., 1999; Chatfield et al., 2005; Matthews et al., 2006; 2004). In an effort to determine the impact of the missing data on our results, we used an extreme sensitivity analysis in which imputed cases missing frailty index data were allocated the highest level of frailty. This sensitivity analysis did not change our results. Further, when we attempted multiple imputation (chained equations algorithm) for frailty index values we found the only predictors of missing frailty index data were related to pathological measures, suggesting that frailty is highly associated with informative dropout in this sample. Our future work will aim to investigate this relationship.

The goal of the cross-sectional regression analyses was to examine the relationship between pathology, frailty, and dementia as close to death as possible, therefore data on frailty and dementia were obtained from the last survey prior to death. This was done to minimize the effect of the autopsy results reflecting worse pathology than was present at the time of the survey from which frailty and dementia measurements were obtained. Even so, the median time from last survey to autopsy was about 2 years and it is likely we were not able to capture terminal decline that would influence frailty. Future investigations should address the issues of terminal decline.

Assessment of neuropathology was not stereological, being based on only one tissue section from each brain area for each staining method. This may lead to under- or over-estimates of pathology in a few cases. However, given the sample size, we assume that the effects of any discrepancies will be minor and cancel out.

Our results are consistent with other reports which show that the vast majority communitydwelling people are not free of neuropathology at time of death (Boyle et al., 2018; Brayne et al., 2009), and neuropathologies typically occur together. In other words, not only are pathological substrates of dementia rarely singular or "pure" in nature but also a "clean" or "unburdened" brain from a pathological perspective is almost unseen in the oldest old (MRC CFAS and CC75C et al., 2012). A few groups have also examined the combined effect of pathology on disease and demonstrated generally, that the more pathology is considered, the better the prediction of dementia (Boyle et al., 2018). While this may not be surprising it is a fact that has been ignored by those seeking a specific treatment for a specific pathology. It is important to consider the combined small effects of all such pathologies as an indicator of the overall health of the system, rather than focus on which one is the most predictive as has been done with the amyloid hypothesis, and now Limbic-predominant Age-related TDP-43 Encephalopathy Neuropathologic Changes (LATE-NC) (Nelson et al., 2019).

Previous work by our groups has shown not only that frailty is associated with biomarkers of Alzheimer's disease (Wallace et al., 2018) but also that the relationship between Alzheimer's-specific neuropathology and dementia changes over levels of frailty and age (Savva et al., 2009; Wallace et al., 2019). While our results were similar in that we see a significant mismatch in neuropathology and dementia status, we did not find an interaction between neuropathology and frailty in relation to dementia status. There are a few differences to take into account when considering the implications of such findings. Perhaps the most influential is the type of pathology measured. The original analysis (Wallace et al., 2019) included very specific "hallmark" features of AD (i.e. plaques and tangles) and examined $\mathrm{AD}$-specific dementia as the outcome, whereas the analyses presented here combine several forms of neuropathology in an index with respect to all-cause dementia as an outcome. The mixed neuropathological index may represent a brainspecific frailty index, and may act as an indicator of overall deficit accumulation in the brain, and thus would not interact with the original frailty index (indicating bodily health) because it would be a reflection of it (with some expected variation).

As our population ages, a growing number of people will live long enough to accumulate several neuropathologies, but many of these people will reach older ages without necessarily experiencing dementia before their deaths. The implications of the current strategies for early detection of specific pathologies is that to "prevent" dementia may actually create more harm than good, in that younger and fitter people will be screened and "disease" will be detected in people who would not have necessarily gone on to develop symptoms. In this way, our work can inform a more public health oriented, preventative approach, by targeting frailty as a means of effective behavioral intervention for dementia risk. We hope this work will inform 
research and clinical approaches in considering dementia as a multi-determined disease that occurs in the aging body, which in essence suggests that the interaction of many mechanisms leading to many diverse pathways that give rise to dementia are likely. Single-mechanism treatments are therefore unlikely to be widely successful, and broad pharmaceutical and non-pharmaceutical therapies such as antiaging compounds (Keller et al., 2019) and exercise (Ahlskog et al., 2011) should be explored more deeply for use in this population.

\section{Conclusions}

The analyses presented here suggest that frailty in its own right contributes to risk for dementia in the oldest old and reduction of frailty can contribute meaningfully to dementia risk. This suggests that future research on frailty interventions should include dementia risk as a key outcome, public health interventions and policy decisions should consider frailty as a key risk factor for dementia (Norton et al., 2014), and biomedical research should focus on elucidating shared mechanisms of frailty and dementia development.

\section{Ethics approval and consent to participate}

Each phase of the CC75C study has been approved by Cambridge Research Ethics Committee. Secondary analyses of these data have been approved by the Dalhousie University/Nova Scotia Health Authority Research Ethics Board.

\section{Availability of data and materials}

The data analysed here are available to be requested from www.cc75c.group.cam.ac.uk.

\section{Conflict of interest}

KR is President and Chief Science Officer of DGI Clinical, which in the last 5 years has contracts with pharma and device manufacturers (Baxter, Baxalta, Biogen, Shire, Hollister, Nutricia, Roche, Otsuka) on individualized outcome measurement. In 2017 he attended an advisory board meeting with Lundbeck. Otherwise any personal fees are for invited guest lectures, rounds and academic symposia, received directly from event organizers, for presentations on frailty. $\mathrm{He}$ is Associate Director of the Canadian Consortium on Neurodegeneration in
Aging, which is funded by the Canadian Institutes of Health Research (CAN-137,794), with additional funding from the Alzheimer Society of Canada and several other charities, as well as from Pfizer Canada and Sanofi Canada (in Phase 1, 2014-2019). The remaining authors have no conflicts of interest to declare.

\section{Source of funding}

We thank all the past CC75C sponsors for financial support spanning two decades (see http://www.cc75c .group.cam.ac.uk/pages/grant/default.htm for full list of project grants) from the Medical Research Council's funding the survey in this analysis to most recently the Abbeyfield Research Foundation, British Medical Association Foundation for Medical Research and General Nursing Council Trust. SH is currently supported by an ARUK Network Support Grant. The funders had no role in the study design, analysis or writing this paper.

\section{Description of authors' roles}

$\mathrm{LW}$ and $\mathrm{KR}$ conceived idea with input from CB. SH prepared and advised on the neuropathological data and analyses and contributed to final draft. LW conceived of design, undertook analyses, interpreted the data, wrote first draft and revised all subsequent drafts. JF advised on methodological design and analysis. OT aided in interpretation and analysis, and revised all drafts. CB is the custodian of the data, and contributed to design, analyses, interpretation, and revised all drafts.

\section{Acknowledgements}

The authors wish to thank particularly the study respondents, their families, friends and the staff in many care homes and collaborating general practices without whose help none of this research would be possible. The current research team gratefully acknowledge the contributions of previous investigators, past research team members, Addenbrooke's Hospital mortuary staff and the helpful comments on earlier drafts of this paper from current collaborators (see full list on CC75C study website: www.cc75c.group.cam.ac.uk/pages/ studypersonnel/default.htm). All neuropathology data was generated by Consultant Neuropathologists at Addenbrooke's Hospital, Cambridge, UK. All slides were prepared by the Cambridge Brain Bank. The Cambridge Brain Bank is supported by 
the NIHR Cambridge Biomedical Research Centre. LW was generously supported by a Mitacs Globalink fellowship which facilitated this collaboration. The authors would also like to thank Emily Zhao for her assistance in data preparation and management.

\section{Supplementary material}

To view supplementary material for this article, please visit https://doi.org/10.1017/S1041610220003932

\section{References}

Ahlskog, J. E., Geda, Y. E., Graff-Radford, N. R., Petersen, R. C. (2011). Physical exercise as a preventive or disease-modifying treatment of dementia and brain aging. Mayo Clinic Proceedings, 86, 876-884. https://doi.org/10 $.4065 / \mathrm{mcp} .2011 .0252$

Anstey, K. J. and Dixon, R. A. (2014). Applying a cumulative deficit model of frailty to dementia: progress and future challenges. Alzheimer's Research \& Therapy, 6, 84. https://doi.org/10.1186/s13195-014-0084-Z

Boyle, P. A. et al. (2013). Much of late life cognitive decline is not due to common neurodegenerative pathologies: cognitive decline. Annals of Neurology, 74, 478-489. https://doi.org/10.1002/ana.23964

Boyle, P. A., Yu, L., Wilson, R. S., Leurgans, S. E., Schneider, J. A. and Bennett, D. A. (2018). Personspecific contribution of neuropathologies to cognitive loss in old age: neuropathologies and cognition. Annals of Neurology, 83, 74-83. https://doi.org/10.1002/ana.25123

Brayne, C. et al. (2009). Neuropathological correlates of dementia in over-80-year-old brain donors from the population-based Cambridge City over-75s Cohort (CC75C) Study. Fournal of Alzheimer's Disease, 18, 645-658. https://doi.org/10.3233/JAD-2009-1182

Brayne, C. et al. (1999). Estimating the true extent of cognitive decline in the old old. Fournal of the American Geriatrics Society, 47, 1283-1288. https://doi.org/10.1111/j .1532-5415.1999.tb07426.x

Canevelli, M., Bruno, G. and Cesari, M. (2017). The sterile controversy on the amyloid cascade hypothesis. Neuroscience E Biobehavioral Reviews, 83, 472-473. https://doi.org/10.1016/j.neubiorev.2017.09.015

Canevelli, M., Cesari, M. and van Kan, G. A. (2015). Frailty and cognitive decline: how do they relate? Current Opinion in Clinical Nutrition E Metabolic Care, 18, 43-50. https://doi.org/10.1097/MCO.0000000000000133

Castell, M. V. et al. (2015). Osteoarthritis and frailty in elderly individuals across six European countries: results from the European Project on OSteoArthritis (EPOSA). BMC Musculoskeletal Disorders, 16, 359. https://doi.org/10. 1186/s12891-015-0807-8

Chatfield, M. D., Brayne, C. E. and Matthews, F. E. (2005). A systematic literature review of attrition between waves in longitudinal studies in the elderly shows a consistent pattern of dropout between differing studies.
Fournal of Clinical Epidemiology, 58, 13-19. https://doi.org/ 10.1016/j.jclinepi.2004.05.006

Clegg, A., Young, J., Iliffe, S., Rikkert, M. O. and Rockwood, K. (2013). Frailty in elderly people. The Lancet, 381, 752-762. https://doi.org/10.1016/S0140-6736(12) 62167-9

EClipSE collaborative members (2009). Cohort profile: Epidemiological Clinicopathological studies in Europe (EClipSE). Fournal of Alzheimer's Disease, 18, 659-663. https://doi.org/10.3233/JAD-2009-1181

Fleming, J., Zhao, E., O'Connor, D. W., Pollitt, P. A. and Brayne, C. (2007). Cohort profile: the Cambridge City over-75s Cohort (CC75C). International fournal of Epidemiology, 36, 40-46. https://doi.org/10.1093/ije/ dyl293

Fried, L. P. et al. (2001). Frailty in older adults evidence for a phenotype. Fournals of Gerontology, Series A: Biological Sciences and Medical Sciences, 56, M146-M157. https://doi. org/10.1093/gerona/56.3.M146

Gallucci, M., Piovesan, C. and Di Battista, M. E. (2018). Associations between the frailty index and brain atrophy: the Treviso Dementia (TREDEM) Registry. Fournal of Alzheimer's Disease, 62, 1623-1634. https://doi.org/10.3233/ JAD-170938

Guaraldi, G. et al. (2019). Compression of frailty in adults living with HIV. BMC Geriatrics, 19, 229. https://doi.org/10. 1186/s12877-019-1247-3

Jack, C. R. et al. (2018). NIA-AA research framework: toward a biological definition of Alzheimer's disease. Alzheimer's E Dementia, 14, 535-562. https://doi.org/10 .1016/j.jalz.2018.02.018

Jansen, W. J. et al. (2018). Age and the association of dementia-related pathology with trajectories of cognitive decline. Neurobiology of Aging, 61, 138-145. https://doi. org/10.1016/j.neurobiolaging.2017.08.029

Keller, K., Kane, A., Heinze-Milne, S., Grandy, S. A. and Howlett, S. E. (2019). Chronic treatment with the ACE inhibitor enalapril attenuates the development of frailty and differentially modifies pro- and anti-inflammatory cytokines in aging male and female C57BL/6 mice. fournals of Gerontology Series A, 74, 1149-1157. https://oi. org/10.1093/gerona/gly219

Lin, C.-K. and Chen, S.-T. (2019). Estimation and application of population attributable fraction in ecological studies. Environmental Health, 18, 52. https://doi.org/10. 1186/s12940-019-0492-4

Livingston, G. et al. (2017). Dementia prevention, intervention, and care. The Lancet, 390, 2673-2734. https:// doi.org/10.1016/S0140-6736(17)31363-6

Matthews, F. E., Chatfield, M., Brayne, C. and Medical Research Council Cognitive Function and Ageing Study (MRC CFAS) (2006). An investigation of whether factors associated with short-term attrition change or persist over ten years: data from the Medical Research Council Cognitive Function and Ageing Study (MRC CFAS). BMC Public Health, 6, 185. https://doi.org/10.1186/14712458-6-185

Matthews, F. E., Chatfield, M., Freeman, C., McCracken, C., Brayne, C. and CFAS, M. (2004). Attrition and bias in the MRC cognitive function and ageing study: an epidemiological investigation. BMC Public Health, 4, 12. https://doi.org/10.1186/1471-2458-4-12 
Mirra, S.S. (1997). The CERAD neuropathology protocol and consensus recommendations for the postmortem diagnosis of Alzheimer's disease: a commentary. Neurobiology of Aging, 18, S91-S94. https://doi.org/10.1016/ S0197-4580(97)00058-4

Mirra, S. S. et al. (1991). The Consortium to Establish a Registry for Alzheimer's Disease (CERAD) Part II. Standardization of the neuropathologic assessment of Alzheimer's disease. Neurology, 41, 479-479. https://doi.org/ 10.1212/WNL.41.4.479

Mitnitski, A. and Rockwood, K. (2015). The rate of aging: the rate of deficit accumulation does not change over the adult life span. Biogerontology, 17, 199-204. https://doi.org/ 10.1007/s10522-015-9583-y

Mitnitski, A., Song, X. and Rockwood, K. (2013). Assessing biological aging: the origin of deficit accumulation. Biogerontology, 14, 709-717. https://doi.org/ 10.1007/s10522-013-9446-3

Mitnitski, A. B., Mogilner, A. J. and Rockwood, K. (2001). Accumulation of deficits as a proxy measure of aging. Scientific World fournal, 1, 323-336. https://doi.org/ 10.1100/tsw.2001.58

Nelson, P. T. et al. (2019) Limbic-predominant age-related TDP-43 encephalopathy (LATE): consensus working group report. Brain, 142, 1503-1527. https://doi.org/10 .1093/brain/awz099

Norton, S., Matthews, F. E., Barnes, D. E., Yaffe, K. and Brayne, C. (2014). Potential for primary prevention of Alzheimer's disease: an analysis of population-based data. The Lancet Neurology, 13, 788-794. https://doi.org/10.1016/ S1474-4422(14)70136-X

Neuropathology Group. Medical Research Council Cognitive Function and Aging Study (MRC CFAS) (2001). Pathological correlates of late-onset dementia in a multicentre, community-based population in England and Wales. The Lancet, 357, 169-175. https:/doi.org/10.1016/ s0140-6736(00)03589-3

MRC CFAS and CC75C, et al. (2012). Impact of less common and "Disregarded" neurodegenerative pathologies on dementia burden in a population-based cohort. fournal of Alzheimer's Disease, 28, 485-493. https://doi.org/10.3233/ JAD-2011-111268

Rockwood, K., Mitnitski, A. and Howlett, S. E. (2015). Frailty: scaling from cellular deficit accumulation? Interdisciplinary Topics in Gerontology and Geriatrics, 41, 1-14. https://doi.org/10.1159/000381127

Rogers, N. T., Steptoe, A. and Cadar, D. (2017). Frailty is an independent predictor of incident dementia: evidence from the English longitudinal study of ageing. Scientific Reports, 7, 1-7. https://doi.org/10.1038/s41598-01716104-y

Savva, G. M., Wharton, S. B., Ince, P. G., Forster, G., Matthews, F. E. and Brayne, C. (2009). Age, neuropathology, and dementia. New England fournal of
Medicine, 360, 2302-2309. https://doi.org/10.1056/ NEJMoa0806142

Searle, S. D. and Rockwood, K. (2015). Frailty and the risk of cognitive impairment. Alzheimer's Research $\mathbb{E}$ Therapy, 7. https://doi.org/10.1186/s13195-015-0140-3

Song, X., Mitnitski, A. and Rockwood, K. (2014). Agerelated deficit accumulation and the risk of late-life dementia. Alzheimer's Research \& Therapy, 6, 54. https:// doi.org/10.1186/s13195-014-0054-5

Sterniczuk, R., Theou, O., Rusak, B. and Rockwood, K. (2015). Cognitive test performance in relation to health and function in 12 European Countries: the SHARE Study. Canadian Geriatrics fournal, 18, 144-151. https://doi.org/10 $.5770 /$ cgj. 18.154

Stoltzfus, J. C. (2011). Logistic regression: a brief primer. Academic Emergency Medicine: Official fournal of the Society for Academic Emergency Medicine, 18, 1099-1104. https:// doi.org/10.1111/j.1553-2712.2011.01185.x

Thibeau, S., McDermott, K., McFall, G. P., Rockwood, K. and Dixon, R. A. (2019). Frailty effects on nondemented cognitive trajectories are moderated by sex and Alzheimer's genetic risk. Alzheimer's Research \& Therapy, 11, 55. https://doi.org/10.1186/s13195-019-0509-9

Trebbastoni, A. et al. (2017). The impact of frailty on the risk of conversion from mild cognitive impairment to Alzheimer's disease: evidences from a 5-year observational study. Frontiers of Medicine, 4. https://doi.org/10.3389/fmed .2017 .00178

Wallace, L. M. K., Theou, O., Andrew, M. K. and Rockwood, K. (2018). Relationship between frailty and Alzheimer's disease biomarkers: a scoping review. Alzheimer's $\mathcal{E}$ Dementia: Diagnosis, Assessment $\mathcal{E}$ Disease Monitoring, 10, 394-401. https://doi.org/10.1016/j.dadm .2018 .05 .002

Wallace, L. M. K. et al. (2020). Neuropathological burden and the degree of frailty in relation to global cognition and dementia. Neurology, https://doi.org/10.1212/WNL .0000000000010944

Wallace, L. M. K., Theou, O., Godin, J., Andrew, M. K., Bennett, D. A. and Rockwood, K. (2019). Investigation of frailty as a moderator of the relationship between neuropathology and dementia in Alzheimer's disease: a cross-sectional analysis of data from the Rush Memory and Aging Project. The Lancet Neurology 18, 10. https://doi.org/ 10.1016/S1474-4422(18)30371-5

Wallace, L. M. K. et al. (2014). Accumulation of nontraditional risk factors for coronary heart disease is associated with incident coronary heart disease hospitalization and death. PLoS ONE, 9. https://doi.org/10 .1371 /journal.pone.0090475

Wilson, R. S., Leurgans, S. E., Boyle, P. A., Schneider, J. A. and Bennett, D. A. (2010). Neurodegenerative basis of age-related cognitive decline. Neurology, 75, 1070-1078. https://doi.org/10.1212/WNL.0b013e3181f39adc 